\title{
A new GTD slope diffraction coefficient for plane wave illumination of a wedge
}

\section{Lumholt, Michael; Breinbjerg, Olav}

Published in:

Proc. of IEEE Antennas and Propagation Society International Symposium

Link to article, DOI:

10.1109/APS.1997.631517

Publication date:

1997

Document Version

Publisher's PDF, also known as Version of record

Link back to DTU Orbit

Citation $(A P A)$ :

Lumholt, M., \& Breinbjerg, O. (1997). A new GTD slope diffraction coefficient for plane wave illumination of a wedge. In Proc. of IEEE Antennas and Propagation Society International Symposium (pp. 1756-1759). IEEE. https://doi.org/10.1109/APS.1997.631517

\section{General rights}

Copyright and moral rights for the publications made accessible in the public portal are retained by the authors and/or other copyright owners and it is a condition of accessing publications that users recognise and abide by the legal requirements associated with these rights.

- Users may download and print one copy of any publication from the public portal for the purpose of private study or research.

- You may not further distribute the material or use it for any profit-making activity or commercial gain

- You may freely distribute the URL identifying the publication in the public portal

If you believe that this document breaches copyright please contact us providing details, and we will remove access to the work immediately and investigate your claim 
91.1

\title{
A new GTD Slope Diffraction Coefficient for Plane Wave Illumination of a Wedge
}

\author{
Michael Lumholt*; Olav Breinbjerg \\ Dept. of Electromagnetic Systems, Technical University of Denmark \\ Building 348, DK-2800 Lyngby, Denmark, Phone: +4545881444 , \\ Fax: +454593 1634, E-mail: ml@emi.dtu.dk, ob@emi.dtu.dk
}

\section{Abstract}

Two wedge problems including slope diffraction are solved: One in which the incident field is a non-uniform plane wave, and one in which it is an inhomogeneous plane wave. The two solutions lead to the same GTD slope diffraction coefficient. This coefficient reveals the existence of a coupling effect between a transverse magnetic (or transverse electric) incident plane wave and the transverse electric (or transverse magnetic) slope-diffracted field. The coupling effect is not described by the existing GTD slope diffraction coefficient.

\section{Introduction}

Slope diffraction is a scattering mechanism contributing to edge diffraction. It occurs when the amplitude of the incident field possesses a non-zero first-order derivative, i.e., a slope, with respect to the distance normal to the plane of incidence. According to the existing GTD solution [1] an incident transverse magnetic (TM) field gives rise to only a TM slope-diffracted field. Likewise, an incident transverse electric (TE) field gives rise to only a TE slope-diffracted field. Here, 'transverse' is with respect to the plane of incidence or the plane of diffraction.

In this work, a new GTD slope diffraction coefficient is derived by solving two different wedge problems. In contrast to the existing coefficient the new coefficient reveals the existence of a coupling effect between the TM incident plane wave and the TE slope-diffracted field and vice versa.

In the first problem the incident field is a non-uniform plane wave given as the superposition of three uniform plane waves. The transverse field components of the non-uniform plane wave are zero at the diffraction point but possess a linear amplitude variation in the direction normal to the plane of incidence and thus a slope. The GTD solution for the wedge illuminated by the non-uniform plane wave is found by superposition of the GTD solutions for each of the three uniform plane waves [2] [3].

In the second problem the incident field is an inhomogeneous plane wave obtained by an analytical continuation of the expression for a homogeneous (uniform) plane wave. The inhomogeneous plane wave possesses an exponential amplitude variation in the direction normal to the plane of incidence and thus a

0-7803-4178-3/97/\$10.00 (C) 1997 IEEE 
slope. The exact solution for the half-plane illuminated by the inhomogeneous plane wave is obtained from an analytical continuation [4]. From a series expansion of the exact field, the contribution to the diffracted field proportional to the slope of the incident field is extracted.

The coupling effect was apparently first reported in [2] for the half-plane case, see also [3]. In a recent paper [5] the existence of the coupling effect is also indicated. The scattering configuration and associated coordinate systems are shown in Fig. 1. A perfectly conducting wedge residing in free space is illuminated by a plane wave. $\theta^{i}$ and $\phi^{i}$ are the polar and azimuthal angles of incidence, $\hat{k}=-\hat{x} \sin \theta^{i} \cos \phi^{i}-\hat{y} \sin \theta^{i} \sin \phi^{i}-$ $\hat{z} \cos \theta^{i}$ the propagation unit vector, $k$ the wave number, and $\zeta$ the intrinsic impedance of free space. The time factor $\exp (j \omega t)$ is assumed and suppressed.

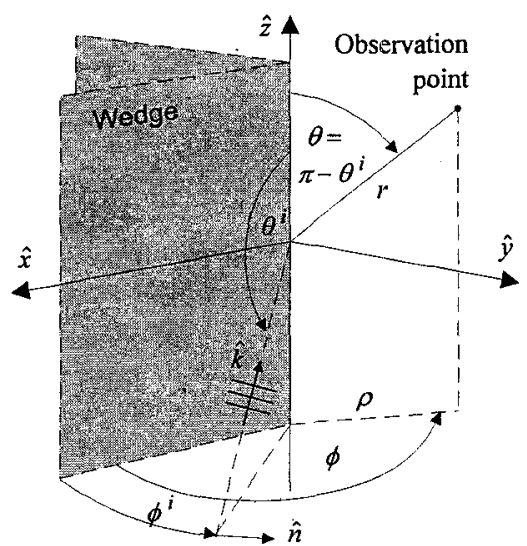

Fig. 1 Wedge illuminated by plane wave.

\section{Non-uniform plane wave illumination}

Two cases exist: quasi-TM illumination and quasi-TE illumination. The quasi$T M$ non-uniform plane wave is expressed in terms of three uniform plane waves:

$$
\vec{E}^{Q T M i}=\frac{1}{j k} \lim _{\epsilon \rightarrow 0} \frac{\vec{E}_{+\epsilon}-\vec{E}_{-\epsilon}}{2 \epsilon}-\hat{\phi}^{i} \frac{E_{0} \cos \theta^{i}}{j k} \exp (-j k \hat{k} \cdot \vec{r})
$$

with $E_{0}$ being a constant and

$$
\vec{E}_{ \pm \epsilon}=\hat{\theta}_{ \pm \epsilon}^{i} E_{0} \exp \left(-j k \hat{k}_{ \pm \epsilon} \cdot \vec{r}\right)
$$

The unit vectors $\hat{k}_{ \pm \epsilon}$ are defined as $\hat{k}$ with the azimuthal angle of incidence $\phi^{i}$ replaced by $\phi^{i} \pm \epsilon . \hat{\theta}^{i} \pm \epsilon$ are the polar unit vectors at $\left(\theta, \phi^{i} \pm \epsilon\right)$. It is emphasized that the limiting sum of the two TM uniform plane waves in (1) will include a $\mathrm{TE}$ uniform plane wave which is non-zero at the edge. Since we want to obtain an incident field being zero at the edge, this TE uniform plane wave is subtracted as the second term in (1). The non-uniform plane wave has the form

$$
\vec{E}^{Q T M i}=\hat{\theta}^{i} E_{0} \sin \theta^{i} \rho \sin \left(\phi-\phi^{i}\right) \exp (-j k \hat{k} \cdot \vec{r})
$$

According to Maxwell's equations the magnetic field is

$$
\vec{H}^{Q T M i}=-\left[\hat{\phi}^{i} \rho \sin \left(\phi-\phi^{i}\right)+\hat{k} \frac{1}{j k}\right] \frac{E_{0}}{\zeta} \sin \theta^{i} \exp (-j k \hat{k} \cdot \vec{r}) .
$$


The GTD field for the non-uniform plane wave illumination is found as the superposition of the GTD fields for the three uniform plane waves. The result is presented in equation (5) below. The case of the quasi-TE non-uniform plane wave is found similarly [2]: the addition of the limiting sum of two TE uniform plane waves and a TM uniform plane wave.

\section{Inhomogeneous plane wave illumination}

The expression for the incident inhomogeneous plane wave is obtained by a complex continuation with respect to the azimuthal angle of incidence $\phi^{i}$ in the expression for the homogeneous plane wave, $\vec{E}_{0} \exp (-j k \hat{k} \cdot \vec{r})$. That is, the real angle $\phi^{i}$ is replaced by the complex angle $\phi_{c}^{i}=\phi^{i}+j \phi_{i m}^{i}$. It should be emphasized that the complex continuation must be applied to the entire expression for the homogeneous plane wave, including the polarisation vector, in order for the inhomogeneous plane wave to satisfy Maxwell's equations. The plane wave relation becomes $\zeta \vec{H}=\hat{k}_{c} \times \vec{E}$, where $\hat{k}_{c}$ is the complex continuation of $\hat{k}$. The real and imaginary parts of $\hat{k}_{c}$ are perpendicular and parallel to the equi-phase planes, respectively. Consequently, there is a field component perpendicular to the equi-phase planes.

In [6] the complex continuation procedure has been applied to solve the wedge problem for normal incidence $\left(\theta^{i}=\frac{\pi}{2}\right)$. In [4] the same procedure has been applied to solve the half-plane problem for oblique incidence.

In this paper the GTD slope diffraction coefficient is derived from the latter solution in three steps. Consider the case of the incident inhomogeneous plane wave obtained from the TM homogeneous plane wave. First, a series expansion in $\phi_{i m}^{i}$ of the incident field is derived. The $O(1)$ term is a TM homogeneous plane wave $\vec{E}_{1}$, and the $O\left(\phi_{i m}^{i}\right)$ term is the sum of a TE homogeneous plane wave $\vec{E}_{2}$ and the quasi-TM non-uniform plane wave $\vec{E}^{Q T M i}(3)$. Second, a nonuniform asymptotic expansion (in $k$ ) of the exact diffracted field is derived and only the dominant terms retained. Third, a series expansion in $\phi_{i m}^{i}$ is derived for the diffracted field. The $O(1)$ term is a TM field which equals the GTD result for $\vec{E}_{1}$. The $O\left(\phi_{i m}^{i}\right)$ term contains only a TM field in spite of the incident TE field $\vec{E}_{2}$. Consequently, the slope-diffracted field due to $\vec{E}^{Q T M i}$ must predict the $O\left(\phi_{i m}^{i}\right)$ TM field and cancel the TE diffracted field due to $\vec{E}_{2}$. This is achieved by the diffraction coefficient in (5).

So far, the work for the inhomogeneous plane wave has been restricted to the half-plane. However, the extension to the wedge is straight forward.

\section{The new slope diffraction coefficient}

The two procedures described in the preceding sections lead to the same GTD slope diffraction coefficient. The coefficient is not valid close to the optical boundaries; for the non-uniform plane wave because GTD has been used to 
obtain the diffracted field, for the inhomogeneous plane wave because a nonuniform asymptotic expansion has been used.

The slope diffracted field becomes

$$
\left\{\begin{array}{c}
E_{\theta}^{d} \\
E_{\phi}^{d}
\end{array}\right\}=\frac{1}{j k \sin \theta^{i}}\left\{\begin{array}{cc}
\frac{\partial D_{s}}{\partial \phi^{i}} & \cos \theta^{2} D_{s} \\
\cos \theta^{i} D_{h} & -\frac{\partial D_{h}}{\partial \phi^{i}}
\end{array}\right\}\left\{\begin{array}{c}
\frac{\partial\left(\vec{E}^{i} \cdot \hat{\theta}^{i}\right)}{\partial n} \\
\frac{\partial\left(\vec{E}^{i} \cdot \hat{\phi}^{i}\right)}{\partial n}
\end{array}\right\} \frac{\exp (-j k r)}{\sqrt{r}}
$$

where $D_{s, h}$ are the GTD soft and hard diffraction coefficients for uniform plane wave illumination and $n=\rho \sin \left(\phi-\phi^{i}\right)$ is the distance from the plane of incidence.

The main diagonal terms in the diffraction matrix are the same as in the existing GTD slope diffraction coefficient. The remaining terms in the diffraction matrix reveal that a coupling exists between the TE incident field and the TM slopediffracted field and vice versa. These coupling terms are of the same order in the wave number $k$ as the main diagonal terms. They vanish in the case of normal incidence $\left(\theta^{i}=\frac{\pi}{2}\right)$.

Furthermore, it is interesting to observe from sections 2 and 3 that a plane wave possessing a slope has a field component perpendicular to the equi-phase planes. It has been shown [4] that the slope-diffracted field may be expressed as the diffraction of this perpendicular component.

\section{References}

[1] P.H. Pathak, "Techniques for high-frequency problems", in Antenna Handbook, Y.T. Lo and S.W. Lee, Eds. , Van Nostrand Reinhold Company, New York, 1988.

[2] O. Breinbjerg, "Half-plane slope diffraction", Tech. Rep. R606, Dept. of Electromagnetic Systems, Tech. Univ. of Denmark, July 1995.

[3] O. Breinbjerg, "A coupling effect in electromagnetic slope diffraction by edges", Proceedings of ISAP'96, Chiba, Japan, pp. 921-924, 1996.

[4] M. Lumholt, "Diffraction of an inhomogeneous plane wave by a half-plane: Exact solution for oblique incidence", Tech. Rep. R650, Dept. of Electromagnetic Systems, Tech. Univ. of Denmark, Jan. 1997.

[5] O.M. Büyükdura, "GTD solution with higher order terms to the diffraction by an edge: towards a uniform solution", IEE Proc.-Microw. Antennas Propag., vol. 143, no. 1, pp. 43-50, Feb. 1996.

[6] R.G. Kouyoumjian, G. Manara, P. Nepa, and B.J.E. Taute, "Diffraction of an inhomogeneous plane wave by a wedge", Proc. IXth Italian Nat. Meeting on Electromagnetics, Assisi, Italy, pp. 53-56, 1992. 